Revue d'histoire de l'Amérique française

REVUE D.HISTOIRE DE L'AMÉRIQUE FRANÇAISE

\title{
La querelle des prisons (Bas-Canada, 1805-1807)
}

\section{Jean-Pierre Wallot}

Volume 14, numéro 1, juin 1960

URI : https://id.erudit.org/iderudit/302030ar

DOI : https://doi.org/10.7202/302030ar

Aller au sommaire du numéro

Éditeur(s)

Institut d'histoire de l'Amérique française

ISSN

0035-2357 (imprimé)

1492-1383 (numérique)

Découvrir la revue

Citer cet article

Wallot, J.-P. (1960). La querelle des prisons (Bas-Canada, 1805-1807). Revue

d'histoire de l'Amérique française, 14(1), 61-86. https://doi.org/10.7202/302030ar d'utilisation que vous pouvez consulter en ligne.

https://apropos.erudit.org/fr/usagers/politique-dutilisation/ 


\section{LA QUERELLE DES PRISONS (BAS-CANADA, 1805-1807) *}

\section{INTRODUCTION}

Une querelle historique apparemment sans importance éclata au cours de l'année 1805. Elle ne semblait impliquer aucun grand principe de race, de langue ou de religion. En effet, la Chambre d'Assemblée se divisa sur la façon de recueillir les fonds nécessaires à l'érection de nouvelles prisons communes dans les districts de Montréal et de Québec. De toute évidence, il n'y avait pas là de quoi alimenter une tempête pendant plusieurs années. Pourtant, d'abord querelle d'intérêts et de systèmes, entre l'intérêt commercial et l'intérêt agricole, entre le système de la taxe directe et celui de la taxe indirecte, elle fit rebondir la querelle de races qui s'était temporairement apaisée. A partir de ce moment, le conflit racial se fera de plus en plus accusé et ne s'éteindra pratiquement plus. Nous avons circonscrit la querelle des prisons aux années 1805, 1806 et 1807. Après cette période, d'autres froissements envenimèrent les susceptibilités nationales déjà irritées des deux groupes ethniques du BasCanada: les Canadiens français et les Canadiens anglais.

Avant d'aborder la querelle elle-même, ses causes et ses répercussions, il nous semble essentiel de décrire brièvement l'état d'esprit des Anglais et des Canadiens de cette période, à la lumière des événements historiques qui l'ont précédée. Dès 1763 , les marchands et les hauts fonctionnaires anglais arrivés dans la colonie nouvellement conquise, s'emparent naturellement des postes-clef dans le commerce et dans l'administration politique. Ils sont d'abord peu nombreux. Mais dirigeant la vie économique et, avec les métropolitains, la vie politique, ils ne permettront jamais aux Canadiens de concrétiser leurs prétentions politiques,

* Thèse présentée à la Faculté des Lettres de l'Université de Montréal pour l'obtention du grade de maître ès-Arts — Montréal 1957. 
ou, plus exactement, leurs prétentions nationales. Déjà sous le régime militaire, ils connaissent leur force; l'exploitation et le développement du British North America, eux seuls peuvent l'accomplir, et ils le savent. Malgré une infériorité numérique qu'ils ne considèrent que momentanée, ils s'installent en maîtres à tous les paliers de l'administration. Or, il arrive que les Canadiens français contrecarrent leur aspiration naturelle à la direction du pays. De là découle l'âpreté de leur haine à l'égard des Canadiens; et ils ne réussiront à tenir vraiment les rênes du pays que le jour où, sous l'Union, ils auront muselé les Canadiens en les mettant en minorité.

Devant l'imminence de la Révolution américaine, Carleton élabore son plan de 1767-1769. Plan de militaire, non de colonisateur, c'est sur ses bases que s'échafaudera l'Acte de Québec. Carleton s'entoure de partisans; et autour de lui s'assemble une équipe que l'on a appelée le «French Party». Ce parti se continuera par la suite, tantôt en majorité, tantôt en minorité, jusqu'à l'Union. Y adhèrent des hommes comme Carleton et Hope, qui ne croient pas à la colonisation anglaise pour diverses raisons. Haldimand rêve d'une vallée du St-Laurent pastorale et idyllique, patrie paisible des seuls Canadiens. Voici donc qu'au désir naturel des Canadiens de demeurer eux-mêmes, vient se fusionner la politique de Carleton. Celle-ci ne peut qu'intensifier cette aspiration inhérente à tout peuple vivant. C'est l'origine de l'illusion longtemps tenace, - elle apparaîtra souvent dans la querelle qui nous occupe - surtout après la constitution de 1791, que le Parlement d'Angleterre protège et appuie les Canadiens français contre les marchands anglais.

A ce parti s'oppose le «British Party 》, composé des marchands anglais de Montréal et de Québec, de la plupart des membres du Conseil législatif et du Conseil exécutif, des hauts fonctionnaires, et fréquemment, du gouverneur. Marriott, un colonisateur de premier ordre en Grande-Bretagne, incarne leur doctrine commune. Il croit en l'assimilation progressive des Canadiens français; il affirme que les marchands assurent la prospérité du pays, que le nombre et l'influence des Anglais augmenteront de plus en plus si les lois sont bien faites et si elles 
favorisent l'immigration britannique et américaine. Il prévoit même que grâce à ses nombreuses richesses naturelles, le Canada deviendra un jour un des grands fournisseurs de l'Angleterre en matières premières. Carleton, devenu lord Dorchester, reviendra au Canada (1786-1787) un tout autre homme: depuis le grand schisme du monde anglais, il veut maintenant faire surgir en Amérique du Nord un immense empire anglais qui puisse rivaliser avec les Etats-Unis. Comme Smith, il croit en l'assimilation des Canadiens. C'est l'époque des grandes offensives du «British Party» (1784 à 1790).

Grâce à un concours de circonstances qu'il serait trop long d'exposer ici, Londres commet en 1791 la bévue de diviser le Canada et d'abandonner pratiquement la Chambre d'Assemblée du Bas-Canada aux Canadiens. Pitt croit que le séparatisme hâtera leur assimilation: admirant la prospérité extraordinaire que connaîtra le Haut-Canada, prévoit-il, les Canadiens voudront réussir autant et s'assimileront. La réalité se situera aux antipodes des espoirs chimériques de Pitt. A cause de sa position géographique et de la présence des Anglais les plus riches et les plus influents dans le Bas-Canada, le Haut-Canada sera à la merci de ce dernier.

C'est dans ce contexte historique que s'insère la querelle des prisons (1805-1807). Jusque là, l'Assemblée du Bas-Canada avait fonctionné sans trop de heurts. Les marchands anglais, conscients de leur puissance économique et frustrés du pouvoir auquel ils aspiraient, rongeaient leur frein, prêts à bondir à l'occasion propice. Ce qui ne les empêchait pas de protester énergiquement et d'expédier des pétitions à Londres à la moindre excuse. Après 1791, quelques Anglais avaient compris la nécessité de l'Union. Mais ces explosions sporadiques n'avaient pas encore empoisonné l'atmosphère de la Chambre. De leur côté, les Canadiens ne pouvaient que croire encore davantage qu'ils constituaient LE groupe national, que Londres leur concédait la maîtrise du Bas-Canada aux dépens des marchands. Pour que la lutte raciale ouverte n'explosât et ne succédât à la lutte plutôt sourde, il ne fallait qu'une étincelle. La querelle des prisons la fournit. 


\section{Chaptrre I}

\section{NÉCESSITÉ DE NOUVELLES PRISONS}

Financement de l'administration avant 1805 - Le gouvernement impérial défraie le gros des dépenses - Premières impositions de droits sur les importations - Etat lamentable des prisons communes à Montréal et à Québec - Représentations du grand jury de Montréal - Représentations de celui de Québec - Nouvelles représentations du grand jury de Montréal.

La première question qui appelle une réponse, au commencement de cette étude, c'est comment la colonie avait-elle financé son administration avant 1805 . En d'autres termes, d'où provenaient les revenus du Bas-Canada. De la Conquête à 1792, le gouvernement impérial avait défrayé le coût des dépenses indispensables à l'administration du Canada. Les seuls revenus qu'il en retirait, au début, venaient du domaine de la couronne qui comprenait les postes $d u$ roi, les forges $d u$ Saint-Maurice, etc...., et de droits d'importation sur certaines marchandises, droits déjà perçus sous le régime français. Simultanément à l'Acte de Québec (1774), le parlement de Londres avait voté un bill ${ }^{1}$ par lequel il imposait un droit de douane sur les eaux-de-vie, les rhums, la mélasse et le sirop importés au Canada, et un droit de «licence » sur les tavernes et les auberges. Le bill contenait aussi certaines dispositions relatives aux amendes et aux confiscations. Les revenus ainsi obtenus devaient servir au maintien de l'administration de la justice et du gouvernement civil.

Dans les discussions qui précédèrent l'adoption de la constitution de 1791, à Londres, des questions fusèrent régulièrement sur la manière dont on allait solder les dépenses publiques sous le nouveau régime. Cependant, il faut admettre que ces préoccupations administratives s'étaient estompées au second plan, derrière les problèmes d'ordre politique. La province ne

1 T. Chapais, Cours d'Histoire du Canada (8 vol., Québec, 1919-1934), 2: 90. A l'avenir: Cours. Cette loi impériale fut désignée sous le titre d'« Acte du revenu du Québec ». 
pourra pas fournir les sommes nécessaires au soutien du gouvernement civil, déclara Adam Lymburner devant la Chambre des Communes; et il exprima l'espoir que le parlement et le gouvernement pourraient endosser ce fardeau pendant quelques années. ${ }^{2}$ De fait, la métropole assuma la plus grande partie des dépenses.

Néanmoins, la colonie contribua elle aussi au budget. Dès sa première session, la législature du Bas-Canada vota une loi établissant «un fonds pour payer des salaires des officiers du conseil législatif et de l'assemblée et pour défrayer leurs dépenses contingentes. » 3 Un droit de douane sur les vins de Madère et autres pourvoyait à ce fonds. Pour l'année fiscale écoulée du 25 décembre 1792 au 5 janvier 1794, le revenu total de la province, incluant le revenu casuel et territorial (domaine du roi, forges, lots et ventes, etc....), les droits de douane et de «licence» imposés par l'«Acte du revenu du Québec», les divers droits imposés par la législature pour rencontrer ses dépenses, les amendes et les confiscations, était de 7,709 louis, ou de $\$ 38,545$. Or, les dépenses du gouvernement civil atteignaient un total d'environ $\$ 125,000$. Le gouvernement impérial devait donc prendre à sa charge la différence de $\$ 86,455 .{ }^{4}$

Durant la session de 1795 , la législature provinciale approuva deux nouvelles lois fiscales. Elles produisirent des résultats plus considérables. L'une consistait en des droits sur le sucre, la cassonade, le café, le tabac en feuille, le sel, les cartes à jouer, ainsi qu'en des droits additionnels sur les eaux-de-vie et les vins. L'autre concernait les colporteurs, en même temps qu'elle augmentait les droits de «licence » sur les tavernes et les auberges. La Chambre d'Assemblée décida d'affecter de façon permanente une somme annuelle de 5,000 louis sterling pris à même le produit de ces impôts, au maintien de l'administration de la justice et du gouvernement civil, pour l'amélioration de la navigation du Saint-Laurent, pour le soutien des maisons de correction et des asiles, etc.... ${ }^{5}$

2 R. Christie, A History of the Late Province of Lower-Canada (6 vol., Québec, 1848-1853), 1:103. A l'avenir: History.

3 T. Chapais, Cours, $2: 91$.

4 R. Christie, History, 1: 152.

5 T. Chapais, Cours, 2: 91. Voir aussi: Statuts provinciaux du BasCanada, vol. I [35, George III, ch. 8]. A l'avenir: Statuts. 
De 1796 à 1804, diverses représentations portèrent à l'attention du lieutenant-gouverneur et de la Chambre d'Assemblée la nécessité d'ériger une nouvelle prison commune dans chacun des districts de Québec et de Montréal. Face aux difficultés de trouver des fonds suffisants pour la construction de ces prisons et de palais de justice, il fut d'abord question d'en créer au moyen de loteries. Mais on ne s'accorda pas sur ce projet. ${ }^{6}$

Entre-temps, malgré divers ravaudages, l'usure et les éléments continuaient à débiffer les prisons. Non seulement les baraques que l'on utilisait comme prisons communes, n'offraient pas la sûreté nécessaire pour claquemurer les prisonniers, mais elles étaient à peine convenables pour des animaux, encore moins pour des hommes. En 1796, à Montréal, après l'évasion de quelques prisonniers, on porta un acte d'accusation contre le geôlier. Le grand jury du district visita la prison. Dans ses représentations aux assises de septembre, le jury déclara que les murs extérieurs du bâtiment étaient si délabrés que non seulement ils ne pouvaient assurer la garde des prisonniers, mais qu'on ne saurait les rendre sûrs autrement qu'en les rebâtissant. ${ }^{7}$

En 1802, le grand jury de Québec rédige de pareilles représentations au sujet de la prison commune de son district. Il entre dans de minutieux détails sur l'état pitoyable du bâtiment. Il nomme les occupants de chaque étage, les uns enfermés sous le coup de condamnations pour crimes graves, comme meurtres, etc...., les autres détenus pour des délits de peu d'importance, mais tous incarcérés ensemble. Et cela, note-t-il, sans compter toutes les souffrances que doivent endurer les prisonniers à cause $\mathrm{du}$ froid en hiver. Il demande enfin au gouvernement d'intervenir pour l'établissement d'une prison convenable où l'on pourrait séparer les prisonniers et faire exécuter les condamnations aux travaux forcés: en effet, les prisonniers ne subissent pas d'autre peine que la détention. "Leurs amis ont le privilège de leur apporter toute espèce de provision, et comme ils sont ainsi

\footnotetext{
6 Douglas Brymner, Rapport sur les Archives publiques du Canada (Ottawa, 1892), xxv. A l'avenir: RAPC.

7 « Représentations du Grand jury de Montréal sur l'état de la prison ... 》, 10 septembre 1796, APC, Q. 97: 93. Traduction: D. Brymner, RAPC (1892), xxv.
} 
dans un état de complète oisiveté, et en constante compagnie d'autres criminels, ils sont en danger de quitter la prison, après l'expiration de leur sentence, plus endurcis dans le vice qu'ils n'étaient lorsqu'ils y sont entrés. ${ }^{8}$

Le 10 mars 1804, dans de nouvelles représentations, le grand jury de Montréal revient à la charge. De l'état de la prison, il brosse un tableau qui, s'il est réaliste, suffit à démontrer la nécessité d'un nouvel établissement; et il n'y a aucune raison de douter de la fidélité du tableau. «La prison actuelle, affirme le grand jury, n'est autre chose que les ruines de l'ancienne prison ravagée par l'incendie; ces ruines ont été réparées tant bien que mal, et si les détenus y sont à l'abri des rigueurs du temps, rien n'empêche qu'ils s'en évadent quand bon leur semble. ${ }^{9} \mathrm{~A}$ cause des nombreux dangers que courent les habitants ainsi exposés à toutes sortes de violences, le jury milite fortement en faveur d'une nouvelle prison. Il prie le tribunal de mettre tout en œuvre afin de trouver une solution.

Le 10 mars 1804, le juge en chef Monk transmit le document au lieutenant-gouverneur, Ryland, en recommandant qu'on y donnât suite. ${ }^{10}$ Invité à soumettre un rapport sur le sujet, le shérif Gray établit que l'édifice actuel était le même qui avait servi sous le régime français, qu'il avait alors et subséquemment ${ }^{11}$ été dévasté par des incendies, et que malgré les réparations récemment accomplies au coût de $£ 615$, il était encore incapable d'assurer la garde des prisonniers. ${ }^{12}$

Devant la concordance de ces témoignages et l'urgence du problème, la législature se décida à passer une loi, en 1805, pour

8 « Représentations du grand jury de Québec sur l'état de la prison $\mathrm{du}$ district...», 17 décembre 1802, APC, Q. 97: 88. Traduction: D. Brymner, Ibid., xxv.

9 « Représentations du grand jury de Montréal sur l'état de la prison...», 10 mars 1804, APC, Q. 97: 96. Traduction: D. Brymner, Ibid., xxv-xxvi.

10 APC, Q. $97: 95$.

11 La prison avait brûlé pour la dernière fois en 1803.

12 Ryland au shérif Gray demandant des explications au suiet des représentations du Grand jury sur l'état de la prison, Québec, 15 mars 1804, APC, Q. 97: 98. Le shérif Gray à Ryland, Montréal, 19 mars 1804, - Explication au sujet de l'état de la prison ...», APC, Q. 97: 99. Aussi: Journal de la Chambre d'Assemblée du Bas-Canada (1805), 66-67. A l'avenir: Journal. 
la construction d'une prison commune dans chacun des districts de Montréal et de Québec. L'Assemblée devait cependant se diviser sur les moyens de taxer à cet effet.

\section{Chaprtre II}

\section{L'ACTE DE LA LEGISLATURE}

La Chambre divisée en deux camps - Débats et votes de la Chambre - Les arguments généraux Adoption du projet de loi présenté par les Canadiens - Texte de la loi — Le bill sanctionné par le Conseil législatif - Débuts de la querelle de races.

Il s'agissait donc de bâtir des prisons. La nécessité d'en construire de nouvelles, tous l'admettaient volontiers. Les divers rapports à ce sujet étaient trop concluants. Mais sur les moyens à prendre pour recueillir les fonds nécessaires à la réalisation du projet, la Chambre d'Assemblée se scinda en deux groupes d'intérêts bien distincts: l'élément agricole, et l'élément commercial qui constituait la minorité. Or, cette division d'intérêts correspondait presque parfaitement au découpage bi-ethnique du BasCanada. Les Canadiens favorisaient l'agriculture et les Anglais, le commerce. Lorsque le conflit inévitable éclaterait entre ces deux avant-gardes, symboliques de la division générale des habitants du pays, il ne pourrait qu'y faire glisser tôt ou tard les deux peuples entiers. L'objet direct de cette querelle n'était d'abord qu'une question de modalités fiscales. Mais, au fond, elle provoquait un duel entre les deux éléments de la population: tout se tient dans une société; et une question de fiscalité ou d'éducation, etc...., se relie toujours à l'ensemble des activités d'une société vivante et organique. Lorsque, sur un même territoire, deux peuples s'affrontent avec les mêmes intérêts et les mêmes aspirations, i.e. devenir une nation, le moindre conflit, à quelque domaine qu'il touche, englobe en dernier ressort ces deux sociétés entières. Pour chacune d'elles, la survivance et l'épanouissement dépendent de la mise-en-tutelle, de l'écrasement ou de l'effritement de l'autre.

Dans le cas qui nous occupe, les deux groupes raciaux coexistent de façon encore assez pacifique. Plus tard, ils seront 
prêts à se ruer l'un contre l'autre et à s'égorger. En défendant l'agriculture, les chefs de la majorité de la Chambre d'Assemblée soutenaient la masse de la population canadienne-française, majoritaire en nombre, minoritaire en puissance, en dynamisme et en richesses. Promoteurs de la cause du commerce, les députés de la minorité plaidaient pour la classe la plus riche et la plus influente. Ils représentaient le groupe anglais qui désirait s'emparer du pouvoir qu'il ne détenait pas encore et qui, quarante ans après la conquête, quarante années d'attente impatiente, leur glissait encore entre les doigts comme une proie évasive; et ce, à cause de leur gouvernement métropolitain et des Canadiens. Les plus riches représentants de cette classe, à Québec comme à Montréal, étaient des Anglais.

Le 6 février 1805, la Chambre délibère sur le rapport du comité de M. de Salaberry. Ce rapport traite de la partie de la harangue du lieutenant-gouverneur qui concernait les prisons de Québec et de Montréal, et des moyens nécessaires pour ériger une nouvelle prison solide et permanente dans chacun de ces districts. Entre autres choses, M. De Salaberry recommande que l'on construise une nouvelle prison dans chacun des districts mentionnés, que la province défraie le coût de ces constructions, que ce coût n'excède pas $£ 9,000$ pour chacune, que cette somme provienne de droits additionnels sur des marchandises importées, etc....

A chaque résolution, sauf en ce qui a trait au principe de la nécessité de nouvelles prisons, la Chambre se divise comme suit, à peu d'exceptions près : ${ }^{1}$

Pour: MM. Fortin, Carron, Alexandre Roi, Brodeur, Weilbrenner, Ferréol Roi, Dumont, Turgeon, Archambault, Raymond, Bourdages, Mondelet, De Salaberry, Perreault, Ross Cuthbert, Rocheblave, Legendre, Grant, Planté, Cartier, Bernier, Vigé, Caldwell, Taschereau, Berthelot, James Cuthbert, Roy Portelance et Bédard. - 28.

Contre: MM. Pyke, Young, Monro, Porteous, Chaboillez, Sir Alexander McKenzie, Richardson et Mure. -8 .

II y a quelques variations chez les Anglais: absences, abstentions, etc.... 
Les autres votes suivent à peu près cette proportion: 24-7, 26-7, 29-3, 26-7, 26-7. ${ }^{2}$ A cette même séance, on charge un comité de 5 membres de préparer et de rédiger le bill. La Chambre confie cette tâche à MM. De Salaberry, Grant, Berthelot, Taschereau et Bourdages, sauf un, tous des Canadiens français.

Déjà se dessinent les deux partis, s'ébauche la division entre la majorité des Canadiens et la minorité des marchands anglais. La proportion moyenne s'établit à environ 26 contre 7 . A chaque proposition, une lutte ardue se poursuit et la minorité soumet des contre-propositions. Un vote les fait avorter. Par le nouveau bill, la Chambre veut imposer des droits additionnels sur les marchandises importées pour subvenir aux dépenses occasionnées par la construction de nouvelles prisons. La minorité anglo-canadienne soutient que multiplier les droits d'importation et reporter les dépenses publiques sur le commerce, c'est paralyser l'essor du commerce et ainsi le développement $\mathrm{du}$ pays tout entier. Taxer la terre, riposte la majorité de la Chambre, c'est enrayer le progrès de l'agriculture, premier fondement de la prospérité générale dans un nouveau pays; d'ailleurs, quel que soit le mode de taxation adopté, la taxe ne sera-t-elle pas payée par les consommateurs ? $^{3}$

Le 22 février 1805, après lecture du projet de loi, Grant propose, appuyé par M. De Salaberry, «que le bill passe et que le titre soit Acte qui pourvoit à l'érection d'une Prison commune dans chacun des Districts de Québec et de Montréal, respectivement, et aux moyens d'en défrayer les dépenses ».

Pour: MM. Turgeon, Roi Portelance, De Lanaudière, Ferréol Roy, Perrault, De Salaberry, Bédard, Bourdages, Cartier, Poulin, Carron, Dumont, Planté, Archambault, Berthelot, le juge Foucher, Rocheblave, Taschereau et Grant - 19.

Contre: MM. McGill, Monro, Richardson, Mure, Sir

Alexander McKenzie, Frobisher et Young - 7. ${ }^{4}$

2 Journal (1805), p. 189 et suivantes.

3 Nous reprendrons ces arguments plus en détails au chap. suivant, alors que nous étudierons les diverses pétitions.

4 Journal (1805), p. 217 et suivantes. 
Pas un seul Canadien français ne vote contre le bill et un seul Anglais se prononce en faveur: Grant. Comme on pouvait s'y attendre, les mêmes députés qui avaient voté pour ou contre les propositions de M. De Salaberry, lors de la séance du 6 février, approuvèrent le bill ou s'y opposèrent. Adopté par la Chambre, il ne restait plus à ce dernier qu'à franchir le Conseil législatif pour devenir loi.

Voici les extraits les plus importants de ce bill:

Acte qui pourvoit à l'érection d'une Prison commune dans chacun des districts de Québec et de Montréal respectivement (25. Mars 1805$)$.

\section{TRES GRACIEUX SOUVERAIN}

ATTENDU que les Prisons communes actuelles des Districts de Québec et de Montréal ne sont point assez spacieuses et ne sont point à d'autres égards bien adaptées pour recevoir et détenir en sûreté les Prisonniers de manière qu'une nouvelle Prison commune pour le District de Québec et une nouvelle Prison commune pour le District de Montréal sont indisperssablement nécessaires; et attendu qu'il a plu graıieusement à Votre Majesté... de donner Votre attention Royale aux Représentations qui ont été faites concernant les dites Prisons, et d'approprier uı terrain dans la Cité de Québec... et un certain autre terrain dans la Cité de Montréal... Il est statué que...

II. [Les commissaires nommés par le gouverneur (I), feront bâtir une prison dans la Cité de Québec et une autre dans la Cité de Montréal] pourvu, toujours, que la somme qui sera déboursée en vertu de cet Acte . . . n'excède pas $£ 9,000$. . . . .

VI. Et afin de défrayer les dépenses qui seront encourrues pour ériger les dites Prisons communes: Qu'il soit donc de plus statué par l'autorité susdite, que depuis et après la passation de cet Acte, il sera levé, perçu, recueilli et payé, pour et sur les Marchandises et Effets respectifs ci-après mentionnés, qui seront importés ou apportés dans aucune partie de cette province, d'aucune place ou places d'où iceux peuvent être légalement importés, et sus et par- 
dessus tous autres Droits maintenant imposés et payables sur iceux dans cette Province, en vertu d'aucun Acte ou Actes du Parlement de la Grande Bretagne, ou du Parlement Provincial de cette Province, les différents taux et droits suivants, c'est-àdire: Premièrement, pour chaque livre, (dite avoir du poids) de thé bou, deux deniers. Secondement, pour chaque livre (même poids) de thé Fouchong ou autres thés noirs, quatre deniers. Troisièmement pour chaque livre (même poids) de thé Hyffon, six deniers. Quatrièmement, pour chaque livre (même poids) de tous autres thés verts, quatre deniers. Cinquièmement, pour chaque Gallon (mesure anglaise) de tous Esprits ou autres Liqueurs fortes, trois deniers. Sixièmement, pour chaque Gallon (même mesure) de toute espèce de Vin, trois deniers. Septièmement, pour chaque Gallon (même mesure) de Mélasse et Sirop, deux deniers. Et suivant ces taux pour aucune quantité plus ou moins grande de tels articles respectivement ...

VIII. Et qu'il soit de plus statué par l'Autorité susdite, que toutes les Marchandises et Effets qui, depuis et après le premier Mai prochain, seront ou pourront ETRE mis à l'Enchère à aucun Encan en cette Province par aucun Encanteur ou Encanteurs, ou par aucune personne qui sera duement qualifiée et aura obtenu Licence... seront adjugés du plus haut enchérisseur et seront et sont par le présent déclarés être sujets à un droit de deux livres dix chellins pour chaque cent livres de la valeur ou prix auquel ils seront vendus .....

IX. [Les personnes licenciées auront seules le droit de vendre des effets, etc. ... à l'encan.]

X. .....la personne ou les personnes qui auront ainsi obtenu Licence comme Encanteur ou Encanteurs, se conduiront et se comporteront bien, vraiment et fidèlement, suivant la vraie intention et esprit de cet Acte...

XI. [Ceux qui vendront des effets à l'encan sans licence, seront passibles d'une amende de $£ 50$, à condition que l'action soit intentée dans les 3 mois qui suivent l'offense.]

$\mathrm{XV}$. [Les encanteurs qui refuseront de rendre compte au Receveur général (XIV) des effets qu'ils 
auront vendus à l'encan, et ce, sous serment, perdront leur licence.]

XVIII. Et qu'il soit de plus statué ... que toutes les Sommes d'Ârgent et droits imposés et exigibles par cet Acte continueront d'être exigibles et payés dans la manière ci-devant ordonnée, pour et durant l'espace et terme de six années depuis le jour de la passation de cet Acte et pas plus longtemps, et s'il arrive que le produit des Sommes d'Argent et droits imposés par cet Acte excède le montant des Sommes d'Ârgent appropriées par le présent Acte pour bâtir les dites Prisons Communes, alors tel surplus restera ${ }^{5}$ et sera réservé entre les Mains du Receveur Général pour la future disposition du Parlement Provincial de cette Province. ${ }^{6}$

Les marchands de Québec et de Montréal n'abandonnèrent pas la partie. Ils présentèrent des pétitions au Conseil législatif contre le bill, ${ }^{7}$ et demandèrent la permission de plaider leur cause devant la Chambre. Le Conseil refusa cette permission et passa le bill à l'unanimité, le lundi 25 mars 1805. Panet, l'orateur de l'Assemblée, s'était adressé au lieutenant-gouverneur en ces termes: «...D'autres objets susceptibles de taxe que ceux qui sont compris dans le bill ont engagé l'attention de l'Assemblée, mais elle se flatte que Votre Excellence sera convaincue que sa conduite sur ce point comme dans les autres occasions, a été dirigée d'après une conviction que les nouveaux Droits imposés sont plus analogues à la situation du pays, et au caractère des

5 Sur la question des surplus possibles, le gouvernement métropolitain se montrera très pointilleux. Presque chaque fois que la question du bill des prisons revient sur le tapis, il ordonne au lieutenant-gouverneur de ne pas utiliser ces surplus sans son autorisation et ses instructions. Voir APC, G. 2:98, Q. 100: 49. «Their Lordships would recommend to His Majesty's Secty of State for the Colonies to instruct the Governor of Lower Canada not to give his consent to any application of such surplus, by Act of Assembly or otherwise, until he shall have received special Directions from His Majesty's government to that effect 》 (Lords du Commerce à Shee, Whitehall, 17 avril 1806, APC, Q. 101(2): 377). "I shall also pay strict attention to your commands in not consenting to any application of such surplus as may arise from the Provincial Act to provide for the erecting a Common Goal..., until I shall have received special directions from His Majesty's government to that effect 》 (Dunn à Windham (no. 12), Québec, 22 août 1806, APC, Q. 100: 241-245).

6 Statuts, [George III, 45 tème année, CAP. XIII] Vol. IV : p. 97 et suiv.

7 Nous étudierons ces pétitions en détails dans le chap. suivant. 
habitants. » Après cette courte introduction, il avait lu le titre $d u$ bill en question. Le Greffier du Conseil législatif prononça alors la sanction royale: « $\mathrm{Au}$ nom de Sa Majesté, Son Excellence le Lieutenant Gouverneur remercie ses loyaux sujets, accepte leur bienveillance et sanctionne ce bill. ${ }^{8}$

Le bill était donc maintenant sanctionné. Dans un même groupe ethnique, la dispute se serait vite volatilisée. Dans le BasCanada, au contraire, la crise devint de plus en plus lancinante. La controverse ainsi amorcée se révéla ardente et passionnée. Elle déborda les cadres de la législature pour rebondir dans des pétitions et dans les journaux. Au Parlement provincial, elle ne manqua pas de sujets pour l'entretenir et l'envenimer. Par exemple, le gouverneur refusa d'augmenter le traitement du traducteur français du nom de P.-E. Desbarats. ${ }^{9}$ La Chambre se sentit visée et considéra ce refus comme une nouvelle marque des mauvaises dispositions du pouvoir envers les Canadiens. Elle venait de se constituer en comité pour étudier la question, lorsque la législature fut subitement prorogée. Ce n'était que jeter de l'huile sur le feu.

\section{ChaptTre III}

\section{LES PETITIONS DES MARCHANDS ANGLAIS}

Lutte acharnée du parti mercantile anglais - Pétition des marchands de Québec et de Montréal Ils prétendent que la loi des prisons va paralyser le commerce - Opinion du procureur général Sewell - Milnes envoie la pétition et divers documents relatifs à la querelle des prisons à Londres - Il donne ses raisons pour avoir sanctionné le bill Pétition des marchands de Londres.

Vaincus à l'Assemblée, les marchands anglais de Québec et de Montréal venaient de perdre la première manche d'un combat qui allait se révéler aussi long qu'acharné. Dès lors, la querelle des prisons devint le symbole de la lutte pour la supré-

8 Gazette de Québec, 28 mars 1805.

9 F.-X. Garneau, Histoire du Canada 8 (9 vol., Montréal, s.d.), 7:55. A l'avenir, Histoire. 
matie du Bas-Canada entre les deux groupes ethniques qui l'habitaient. De ces escarmouches s'établirait tôt ou tard une relation hiérarchique entre eux. Cette première obstruction heureuse de la part des Canadiens français fit comprendre encore plus clairement aux marchands les dangers de la constitution de 1791 qui accordait virtuellement la domination de la Chambre à ceux qu'ils voulaient dominer et angliciser.

Après le vote du bill et le refus du Conseil législatif de leur laisser plaider leur cause, les marchands de Québec et de Montréal firent parvenir des pétitions au lieutenant-gouverneur lui demandant de réserver le bill au bon plaisir de Sa Majesté. Le roi, en effet, pouvait apposer son véto à toute loi votée dans la colonie durant une période de deux ans suivant son adoption et sa sanction royale dans la province. Par ces pétitions, le parti mercantile espérait bien faire rappeler la loi à Londres. Il savait quel ascendant il avait exercé de tout temps sur l'Angleterre: le commerce n'était-il pas le sang de cet immense corps, de ce colossal empire qui avait haussé l'Angleterre au premier rang des puissances mondiales ? Et comme le sang afflue à la tête et nourrit le cerveau, les commerçants n'influençaient-ils pas le pouvoir politique et n'en étaient-ils pas les maîtres pour une bonne part? Le parti mercantile arc-boutait et vivifiait tout l'empire, ce cycle vital métropole-colonie, colonie-métropole, véhicule d'échanges constants de cadres politiques, de capitaux, d'hommes, d'idées, etc...., composant un tout indissociable et organique qu'on ne peut tronquer sans danger pour tout l'organisme.

En février 1805, les marchands de Montréal envoyèrent une pétition ${ }^{1}$ au Conseil législatif contre le projet de loi voté par la Chambre. Vers la même date, les commerçants de Québec expédièrent une reproduction ${ }^{2}$ de la pétition des Montréalais.

Les marchands affirment d'abord que le système de taxation indirecte tel que préconisé par la Chambre d'Assemblée établit «a precedent in direct opposition to sound practice under the British Constitution, and fraught with every temptation to

\footnotetext{
$1_{\ll}$ Petition des marchands de Montréal au Conseil législatif ...», Montréal, février 1805 , APC, Q. 99: p. 100 et suivantes.
} 
waste and profusion of the public monies by drawing them trom the general purse to be expended upon objects of a local nature $\gg .^{2}$

Avec l'introduction de la loi criminelle anglaise dans le BasCanada, disent-ils, il faut aussi que l'on pourvoie à la construction des prisons de districts ou de comtés aux frais de ces districts ou comtés. C'est la pratique en Angleterre où l'on semble accepter le principe que pour les édifices, routes publiques et autres buts d'utilité et d'économie locales, une taxe spéciale est imposée à chaque district. Au contraire, pour ce qui a trait à l'administration de la justice et le soutien du gouvernement civil de cette province, les taxes devraient alors affecter les importations de marchandises.

Connaissant la jalousie naturelle de la métropole pour ses droits, sa crainte ${ }^{3}$ d'un gouvernement colonial trop indépendant et son mercantilisme normal, les pétitionnaires soulignent le fait que ce principe de taxer le commerce, appliqué à des fins locales, «appears a dangerous exercise of Power, and tends to weaken the connection with the Mother Country, by an indirect attack upon her reserved right, to regulate the general Trade of the Empire with the Colonies as it may hereafter be applied to discourage the Importation of British Manufactures in order to encourage such as are local; that as a Colony of Great Britain, it became us to avoid such dangerous, and objectionable methods of raising Money..., when the inhabitants of the Districts respectively, are sufficiently able by Constitutional means to defray the Expense of these and other Objects of a local nature ».

2 《Petition des marchands de Québec...»», Québec, [sans date] APC, Q. 97: p. 101 et suivantes. Nous utilisons le texte de la pétition des marchands de Québec, qui est une copie de la précédente.

3 Cette crainte n'est pas nouvelle et date surtout de la Révolution américaine. Le $1^{\text {er }}$ novembre 1800 , Milnes écrivait au Duc de Portland: « ceux qui portent intérêt à l'administration considèrent que c'est une circonstance heureuse que le revenu ne soit pas égal aux dépenses... il y a lieu de croire que si on pouvait amener la province à s'imposer des taxes à peu près égales aux besoins du gouvernement exécutif, l'Assemblée réclamerait probablement le droit de réglementer et le contrôle sur tout, ce qui ne manquerait pas de produire les conséquences les plus préjudiciables pour le gouvernement de la colonie, en le mettant dès ce moment à la merci des volontés d'une assemblée populaire », i.e. de Canadiens français. (APC, Q. 85: 229). 
Puis, les marchands brandissent devant les autorités le spectre d'une contrebande possible avec les Etats-Unis. Or, sous le régime anglais comme sous le régime français, une telle contrebande ne peut à la longue que profiter au commerce du plus fort et désagréger l'économie de la colonie qui veut justement grandir et exister par soi, indépendante des Etats-Unis: «... A contreband Trade is now carried on in Dutiable Goods, to a considerable extent, and therefore to impose further Duties upon Importation without necessity, is to encourage illicit trade.»

D'ailleurs, dans le Haut-Canada, n'a-t-on pas réussi à aplanir les difficultés et à régler le problème par des moyens locaux ?

Ces nouvelles taxes rapporteront environ dix mille livres par année sur la moyenne d'importation des articles en question, soit $60 \%$ de toutes les taxes ensemble imposées par la législature sur les importations, «a circumstance which must strike every thinking man with astonishment, more especially, when the object to which this sum is to be applied, and the duration of the said duties are taken into consideration ».

Les marchands cherchent ensuite à repousser l'objection que ce sont les consommateurs qui, finalement, paieront ces nouveaux droits :

The different duties, levied thereon by Acts of the British Parliament, and the Provincial Statutes go fully to $£ 25,000$ yearly exclusive of those usually denominated King's Duties, which may amount to two thousand pounds more, so that on a few Articles of Commerce, Duties are already levied which exceed five per centum on the Aggregate value of all Importations, and now it is proposed for local purposes to add Ten thousand pounds more, all which must in the first instance be advanced by a few merchants and perhaps lost to them for ever, for altho' it is an opinion held by some speculative Writers, that the Consumers must, and will ultimately pay the Duties so advanced, yet experience often dearly bought has demonstrated the contrary, and that the Sale price is regulated by the quantum of the Commodity at Market, with the demand for it... On the average of Seven years past, the Duties levied on Articles liable thereto in this Province, have been lost by 
the Importers and when it is considered that from the nature of the Climate... we are shut out from all Communication by Sea for Six months in the Year, and consequently prevented from re-Exportation so as to keep up the level between quantum and demand; as in other countries is practised; they trust that it will appear, that the Importer must sell at the price of the Canada Market, whatever it is, and therefore the Taxation of Commerce even for general purposes, should be done with a sparing hand, but for all purposes whatever as the principle of the bill involves, is imprecedented and oppressive.

Même s'ils ont accepté de bon cœur les taxes justes dont le produit devrait desservir le bien commun, les marchands ne peuvent souscrire en silence à tout impôt « which the false doctrine of Taxation by expedience at the sacrifice of constitutional principles may suggest ».

On a rarement recours à une taxe temporaire sur le commerce, sauf dans des cas très urgents ou pour la protection du commerce en général. La raison en est que ces taxes temporaires «place the Merchant paying and the Merchant escaping them, upon a very unequal footing, by exposing the former when the Taxes cease, to be met by the latter with a quantity of similar Goods, not liable to pay such Taxes. Taxation on Commerce for objects of temporary expense is therefore also objectionable on that Ground, and to continue to lay Duties, after the purpose for which they were intended is attained becomes doubly so ».

Puis, ici, les pétitionnaires rappellent qu'au Canada, comme dans toute colonie, les marchands locaux s'approvisionnent en capitaux et en marchandises auprès de fournisseurs et de marchands de la métropole. Un commerce colonial ne peut s'édifier et s'accroître que s'il se greffe sur la métropole. (Ce qui explique la déchéance du commerce canadien. Il n'a pas survécu à la Conquête et est mort après quelques soubresauts: privé des cadres impériaux de la France, il ne s'abreuvait plus à sa source. Il ne pouvait que dépérir, comme la branche émondée du tronc). Cette taxe «appears... as virtually Taxing the British Merchants, for there being few importers in this Province, who carry on Trade on their won Capital, but on the contrary on 
the Credit they obtain from Great Britain, and that the Duties must at all events be paid it follows that unless such goods are sold, so as to cover their Cost with all charges and Duties inclusive, whatever they may fall short thereof is in fact so much taken from the Original British Creditors of such Importers ».

Les marchands concluent sur une note un peu ironique. Si les Canadiens français réussissent à se construire autant d'églises, disent-ils, pourquoi ne pourraient-ils pas payer pour l'érection des prisons :

No excuse of poverty in the Land Holders of this province can possibly be offered as a reason for Taxing Commerce in all cases and thereby exonerating the Proprietors of Lands from all Burthens, even for their own security, it being a well known fact, that in the Districts of Quebec and Montreal and of Three Rivers also, every Church which has been built for many years past has cost from Two to Five thousand pounds Currency. And at the expense of the inferior Land. Holders or Censitaires, in each of the Parishes respectively, in comparison with this, a temporary Assessment on fixed Property in the District of Quebec which contains Fifty Parishes, and in that of Montreal which contains above Sixty, would bear very light indeed ...

(Signed)

$\begin{array}{ll}\text { Geo: Allsopp } & \text { Ias. Gray } \\ \text { Angus Shaw } & \text { Peter Brehaut \& Co. } \\ \text { Lester and Morrogh } & \text { Blackwood, Paterson \& Co. } \\ \text { Irvin, Macnaught \& Co. } & \text { Burns \& Moolsey } \\ \text { Mourd \& Bell } & \text { Sarjeant \& Downs } \\ \text { John Mure } & \text { Jones \& White } \\ \text { Jn' Painter } & \text { J. Salmon } \\ \text { John Stewart \& Co. } & \text { John Jones } \\ \text { George Symes } & \end{array}$

Les pétitionnaires demandent donc le rappel de la loi et apposent leur signature au document. Parmi ces représentants du grand commerce, on ne décèle aucun Canadien.

Sur réception de la pétition, Sir Robert Milnes demanda l'avis du procureur général. Malgré ses affinités avec le « British 
Party » et ses visées clairement anglicisatrices, ${ }^{4}$ Sewell répondit qu'au point de vue légal, il ne croyait pas le bill inconstitutionnel. $\mathrm{Vu}$ l'urgence de la situation, il conseilla même au gouverneur de le sanctionner, quitte à laisser aux conseillers du roi le soin de trancher la question:

..... I confess I have not hitherto, nor do I now conceive that it [l'Acte] is unconstitutional.

Gaols in England are undoubtedly built and repaired at the expense of each County, but this is in consequence of a positive Statute. By the Statute 23' Henry VII c. 2 in Essex, and several other Counties, the Justices of Peace were empowered to erect new Gaols, and to assess the Inhabitants of each County respectively to discharge the expense thereby to be incurred. This Statute which was temporary was afterwards continued by the 5 Elir. c. 24 and 13 Elir. 25 and by the 11 \& 12 Will: III c. 1q. The substance of it was re-enacted, with respect to all the Counties in England, with several additions... and finally made perpetual by the 6 Geo. III c. 1q.

But the statute of the 23 Henry VIII c. 2 expressly provinded, that the Gaols which it authorised to be erected by a local Tax on each County should nevertheless « once made» be « repaired and amended 》 at the general Expense « by allowance to the Sheriff out of the Exchequer $\gg$ and this continued to be the Law of England until by the Statute $11 \& 12$ Will: III c. 1q., it was enacted that the Common Gaols of each County should not only be erected, but repaired by assessment on the Inhabitants of the County. The Gaols ... were erected at the common charge of the Kingdom, until it was though expedient to change that system. [Or, au Québec, il n'y a pas de statut comme en Angleterre qui empêche de taxer le tout ou l'ensemble de la province pour l'érection de nouvelles prisons]. I am further of opinion, that it will be most adviseable for Your Excellency to pass the Bill; in my mind there appears no sufficient cause, to justify your Excellency in rejecting the supply

4 Sewell sera l'instigateur de la première tentative de correction de la constitution de 1791 . Son plan de 1810 proposera de peupler le BasCanada d'Anglais et d'unir les deux Canadas pour mettre les Canadiens français en minorité. 
granted to His Majesty by the Bill, on the contrary Gaols in both Districts, but in the District of Montreal in particular, are on indispensable and immediate necessity; and if upon consideration the Taxes mentioned in the Bill, should be thought by His Majesty's Ministers, to be unconstitutional or inexpedient, it may be disallowed, the Act of the $31 \mathrm{Geo}$ : III c. 31 sec. 31 having provided, that His Majesty may disallow any Bill at anytime, within two years after its reception by the Secretary of the State.

(signed)

\section{J. Sewell}

att. General

Quebec, 23 March 1805. ${ }^{5}$

Le Conseil législatif ratifia le bill le 25 mars. Le 12 avril, dans une dépêche à Londres, Milnes rend compte des débats qui se sont déroulés à la Chambre d'Assemblée et étale ses propres raisons pour avoir sanctionné le bill. Il y inclut aussi une série de documents relatifs à la querelle des prisons; copie de l'Acte, représentations diverses du grand jury de Montréal et de celui de Québec, la pétition des marchands de Montréal et de Québec, le rapport $d u$ procureur général Sewell, etc....

Milnes insiste d'abord sur l'insuffisance des maisons de correction actuelles et sur la nécessité de nouvelles prisons. En 1803, rappelle-t-il, le feu a rasé partiellement la prison de Montréal et malgré les réparations subséquentes, les murailles ne sont pas sûres. Le bill a provoqué bien des débats à la Chambre: «...The House was unanimous with respect to the necessity of a Provision for building Gaols in such of the Districts of Quebec and Montreal, but differed upon the Ways and Means: those Members who are concerned in the Trade of the Province were urgent for a local Assessment on the Land; but the Land Holders, both English and Canadian, were of opinion that the Sum required for this Purpose ought to be raised by Duties imposed by the Legislature $\gg .7$

5 \& Rapport du procureur général Sewell sur l'Acte concernant la construction de prisons ... 》, Québec, 23 mars 1805, APC, Q. 97: 109.

6 Par les votes à la Chambre d'Assemblée, tels que donnés au chapitre précédent, nous savons qu'il n'y avait qu'un ou deux Anglais en faveur du bill adopté par la Chambre.

7 Milnes à Camden (No. 22), Québec, 12 avril 1805, Q. 97: 84-109. 
Il a reçu une pétition signée par un grand nombre de marchands qui lui demandaient de réserver le bill pour raison d'inconstitutionnalité. Il envoie une copie de cette pétition et du rapport du procureur général sur la pétition avec sa recommandation que le bill passât.

Lui-même a ratifié le bill au nom du roi pour diverses raisons :

.... It was argued in behalf of the Land Holders, and in justification of their objections to an Assessment, that out of the feudal Profits or Revenues arising from the Royal Domain, the Crown of France was held to provide Gaols and Courts for the administration of justice in Canada; these Revenues (so appropriated heretofore) have, since the establishment of the present Constitution, gone towards defraying the general Expenses of the Civil Government, as therefore it has had the use of that Fund for all purposes generally the Advocates of the Bill contend, that the Province generally should contribute towards the erection of any Goal which may be wanted: as the Revenue arising from the feudal Rights, which has already been appropriated to general Purposes, is more than equivalent to what is intended to be raised by the present Act; and however the judicial System may have been altered and improved by the introduction of the Criminal Law of England, the Land holders contend that the Revenue Arising to the general Fund from the above source should still be considered as relieving those that pay it from any extraordinary Contribution beyond what may be furnished by others of His Majesty's Subjects equally interested in and benefitted by the administration of Justice.

D'ailleurs, des précédents étayent amplement l'établissement de taxes générales pour des fins locales: l'Acte qui pourvoit à défrayer le coût des cours de justice de Québec et de Montréal, les taxes pour la maison de correction de Montréal, les réparations de la prison et la construction de ponts. Et puis, poursuit Milnes, le commerce du pays peut très bien se charger de cette nouvelle taxe, surtout que le poids en affectera d'abord les consommateurs. "The only objection therefore which appears 
to me of Weight is, whether the additional Imports may not be injurious to the Merchant in England, a Question that can only be determined in England. » En d'autres termes, ce sera au gouvernement anglais de juger si l'Acte doit être rejeté. Milnes ajoute que la majorité des deux Chambres a favorisé le bill.

Après ces considérations d'ordre juridique, Milnes donne son opinion véritable sur le sujet, bien qu'à mots couverts. Il aurait préféré une taxe comme en Angleterre. Mais malgré l'appui qu'accordent les membres les plus respectables et les plus fidèles de l'Assemblée, i.e. les Anglais, à la taxe directe, la majorité de l'Assemblée, i.e. les Canadiens français, ne semble pas disposée à accepter ce principe pour le moment: "With respect to an Assessment on Land, though I should have though it highly desirable, as establishing a Precedent that any future period might be adopted for other local Purposes, I must say I never had any hope that the Measure could be carried, though it was proposed by some of the most respectable Members, and those who have on all occasions shown the most steady attachment to His Majesty's Government, but the Bias against this Mode was so strong, and has been so much encreased by the late Dissension on the Subject, that it is my decided opinion no further attempt of the kind ought to be made at present, as I am confident it would be found impossible to contend with the late Majority of both Houses $\gg .^{8}$

Mieux vaut accepter l'Acte tel que voté: «The Burthen are called for by an imperious Necessity, are the only means which the Prejudices of a conquered People will admit, and are imposed for a matter... deeply interesting to the Protection and Safety of the Government itself ». Ces diverses expressions - «Bias », «Prejudices of a conquered People » - aiguillent clairement la sympathie de Milnes à la cause des marchands. Plus que cela,

8 Milnes écrira aussi dans une autre lettre: «It may not however be altogether unimportant to observe further that was the Sum at present required for those purposes to be raised by an Assessment on Land throughout the Two Districts, the same Landholders, residing without the particular Counties of Quebec and Montreal might be called upon a second time for an Assessment whenever the Population increases sufficiently to make the establishment of County Gaols necessary in this province 》 (Milnes a Camden (no. 26), Québec, 11 juin 1806, APC, Q. 97: 146). 
il camoufle mal la rancœur et l'amertume qui sourdent en lui, les mêmes que celles des commerçants: Ils sont les maîtres, mais ils doivent se déjeter devant les «préjugés des conquis 》, à cause de l'urgence de la situation et du fait que les marchands peuvent très bien soutenir cette nouvelle charge.

Entre-temps, voulant faire jouer en leur faveur la puissance économique et politique de leurs fournisseurs métropolitains, les marchands de Montréal et de Québec leur avaient demandé d'exercer des pressions auprès du gouvernement anglais pour le rappel de l'Acte. Ils espéraient que ce dernier céderait aux pressions simultanées des commerçants de Londres et de ceux du Canada. Les métropolitains envoient donc une pétition au ministre des colonies par l'entremise d'un M. Inglis, de la maison Phyn, Inglis \& Co. En général, ils reprennent les mêmes arguments que les coloniaux; toutefois, ils insistent davantage sur le tort que cette nouvelle taxe pourrait infliger au commerce de l'empire:

.... Your memorialists therefore for themselves, and in behalf of their correspondants, beg leave to call the attention of your Lordship to the subject, in the Hope that Your Lordship upon consideration of the principle of the Act and its tendency to injure the Trade of the Mother Country and her West India Colonies, as well as the Canada, will be pleased to advise His Majesty to signify his pleasure that it may not take effect.

In their opinion, the principle of taxing the general Trade and Commerce of the Colony for local purposes, is impolitic and at variance with the practice of the other British Colonies, and inconsistent with the general system and spirit of British Legislation. They beg to submit... whetter every encouragement ought not in good policy to be given to promote Mutual Intercourse and advantage, and that if this Act take effect whether it will not be inconsistent with the Principle of the Act of the British Parliament passed in the Twenty Eighth year of His present Majesty, intituled an Act to allow the Importation of Rum or other Spirits from His Majesty's Colonies or Plantations in the West Indies into the 
Province of Quebec without payment of Duty under certain conditions and Restrictions. ${ }^{9}$

Loin de favoriser le commerce britannique, cet acte entrâ̂nera la contrebande avec les Etats-Unis, le puissant voisin de la colonie; ce qui en fera d'autant moins pour l'empire:

.... It will encourage the American Traders to rival British Subjects in their Intercourse \& Commerce with the Indians, and above all, it is to be apprehended that it will occasion a Misunderstanding between the Provinces of Upper and Lower Canada by compromising the Interest of the former which most assuredly ought not in justice to be made to contribute in the smallest degree to the expense, [puisque le Haut-Canada, nouveau et pauvre comme il est, a construit de tels édifices au moyen de taxes sur les terres].

Enfin, les pétitionnaires accusent les Canadiens de partipris: ces derniers, disent-ils, sont bien aises d'imposer des taxes sur le commerce et sur les vrais colonisateurs du pays, les Britanniques, puisqu'eux-mêmes sont pauvres et ne commercent pratiquement pas. D'ailleurs, s'ils peuvent se payer le luxe de tant d'églises catholiques, ils peuvent défrayer le coût de prisons communes:

That it appears to Your Memorialists, that the Majority of the Legislature of Lower Canada being composed of the new or French Subject, seem unwilling to impose any direct or immediate tax on their fellow Subjects. Even for any local purpose, and carrying on themselves no extensive Commerce, but deriving their means of support chiefly from landed property, do not sufficiently consider the evil tendency of the Burthens and Restrictions laid on the Trade, or of the duties they impose on it, which in the first instance, must be paid by their British Importer, thereby materially affecting the Remittances of Your Memorialists. Your Memorialists understand that in Lower Canada, it has been the practice to build the Roman Catholic Churches by Assessment in each parish and that in the

9 Inglis à Castlereagh, Londres, 27 novembre 1805, Mémoire des marchands de Londres, APC, Q. 99: 285-289. 
Districts of Quebec and Montreal there are, according to their information about one hundred Churches, which upon an average cost about two thousand pounds each, it cannot therefore in their opinion be oppressive to erect two Jails in the same districts, by the like means, especially as the British Inhabitants will contribute to which they are not liable in the Case of Churches...

(signed)

Risdale, Hamilton \& Co.

McTavish, Fraser Thomp. \& Co.

Gray, Freeman \& Co.

John Auldyo

Inglish, Ellice \& Co.

Brickwood, Daniell \& Co.

Mackenrys, Gillespie,

Parker \& Co.

Ainsi, la réaction ne s'était pas fait attendre. Elle avait jailli, spontanée, de tous les représentants du commerce, tant dans la colonie que dans la métropole. Culbutés à la législature, les marchands coloniaux se débattent de toutes leurs forces. Ils ne peuvent tolérer la sujétion à la majorité canadienne-française, formée de «pauvres et d'ignorants», titres dont ils les affubleront trop souvent. Eux, les marchands, sont les plus riches, les plus puissants, les vrais colonisateurs. Ils veulent piloter la barque. Ils porteront la lutte jusque dans leurs réunions publiques et dans leurs journaux, ce qui déclanchera des réactions non moins vives de la part des Canadiens. En lui-même, le simple bill des prisons ne peut justifier la violence et l'aigreur de la querelle. C'est que déjà il était devenu le prétexte à une épreuve de puissance entre les deux éléments de la population du BasCanada.

(à suivre)

JEAN-PierRe WALLOT, B.A. 